\title{
Parental Population Sizing in Evolutionary Strategies
}

\author{
Tao-Yuan Huang \\ Institute of Electrical Engineering. \\ National Taiwan University, \\ Tiapei, Taiwan, ROC, 106 \\ hubert(d)ipmc.ee.ntu.edu.tw
}

\author{
Yung-Yaw Chen \\ Institute of Electrical Engincering, \\ National Taiwan University, \\ Taipei, Taiwan, ROC: 106 \\ yychen(o)ipmc.ee.ntu.edu.tw
}

\begin{abstract}
One of the major differences of Evolution Strategies (ES) from other Evolutionary Computation (EC) is self-awareness of the distinction between parental and offspring populations. This feature is all the more prominent when it comes to the availability of two ES variants: COMMA and PLUS ES (i.e. $(\mu, \lambda)$ and $(\mu+\lambda)$ ES). Even though these two schemes capture, respectively, the antipodal emphases on exploration and exploitation, conventional practices of ES still suffer from the problem of premature convergence. By the proposition of an adaptive parental population sizing approach, the authors wish to buttress the original ES scheme. This population sizing approach differs from previous ones in that only size of the parental population is adjusted. Simulation on several benchmark problems vindicates the virtue of this modification. Moreover, the modified algorithm also sheds light on convergence status of the population for a given optimization task, hence allowing further improvements on ES toward premature convergence checking and parallel search mechanisms.
\end{abstract}

\section{Introduction}

Evolution Strategies (ES) were developed by Rechenberg [1] and Schwefel [2] to solve difficult parameter optimization problems. Like Genetic Algorithms (GA) [3] and Evolutionary Programming (EP) [4], this methodology falls into the generic discipline of Evolutionary Computation (EC). EC are biology-inspired techniques most often employed for optimization and search problems. The rationale behind these techniques is fitness-driven random walk after a (global) optimum by an evolving population of possible solutions. Over generations, the population improves in fitness values with search directions and convergence velocity determined by evolution operators. A typical evolution cycle can be summarized into the loop shown in Figure I (after the figure given in [5]). As can be observed, recombination (crossover), mutation, and selection are all common evolution operators among different EC branches.

The performance of these population-based algorithms depends greatly on interaction between evolution operators and the optimization problem at hand. The operators inclination shapes the virtual fitness landscape as seen by the population, while this sampled terrain leads the direction of subsequent search. This complication often results in a prolonged search process or even premature convergence to some local optimum. Moreover, the interactive nature of these operators also makes it very difficult to hand-tune EC. for the wide array of problems. In recognition of this fact, adaptive evolutionary operators are studied and implemented.

Among these researches, the self-adaptive mutation mechanism in ES presents itself as a promising contrivance. It is assumed that strategy parameters for surviving individuals are better than those perish. Under this assumption, the self-adaptive mechanism should be able to moderate the degree of mutation for each individual according to its proximity to the (global) optimum. However, real-world implementations of ES still suffer from slow convergence or even premature convergence due to a lack of coordination among its evolutionary operators. This difficulty results mainly from: (1) blind belief in the spontaneous correspondence firom self-adapted mutation step sizes to local fitness terrain, and (2) greediness of the ranking-based selection which erase the trace of interaction between mutation and problem surface. This paper tries to address the second shortcoming by adjusting dynamically the size of the parental population from generation to generation. Not only do simulation results vindicate the virtue of this modified ES. the evolution behavior also demonstrates interesting links between population diversity measures and solution convergence.

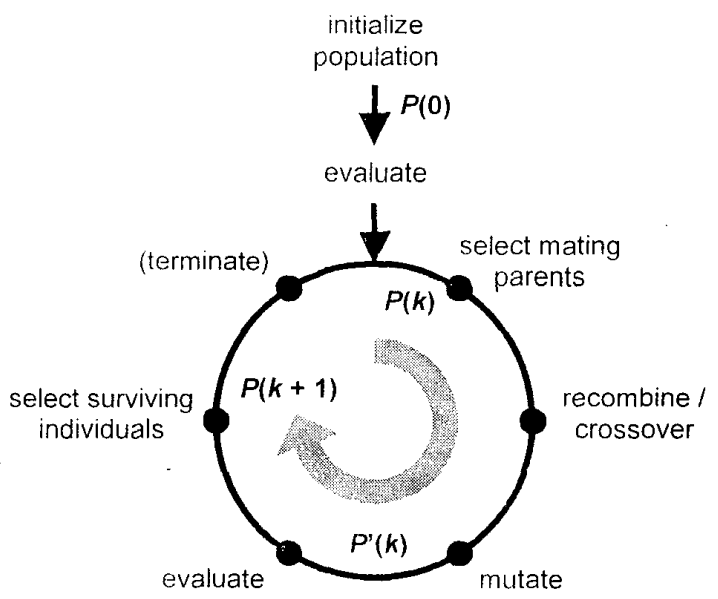

ligure 1. I Generic LC Evolution (ycle (atter |5]) 


\section{Evolution Strategies}

The Evolution Strategies used in this paper are as those described in [6 $6 \| 7]$. Each run of ES computation starts with a randomly generated initial population $P(0)=\left\{\vec{a}_{1}(0), \cdots, \ddot{a}_{n}(0)\right\}$ of ${ }_{\mu}$ individuals.

Definition 1. Individual in an ES Population $\left(a_{j}(k)\right)$

For standard ES cmploying n-dimensional uncorrelated self-adaptive mulation over an n-dimensional optimization problem, the ES population at the $k^{\text {th }}$ generation is denoted as

$$
P(k)=\left\{\vec{a}_{1}(k), \cdots, \ddot{a}_{n}(k)\right\}
$$

in which the $j^{\text {th }}$ individual is of the form

$$
\vec{a}_{j}(k)=\left(\vec{X}_{j}(k), \vec{\sigma}_{j}(k)\right)
$$

where

$$
\vec{X}_{i}(k) \in R^{n}
$$

is the vector of object variables in the $n$-dimensional search space, and

$$
\ddot{\sigma}_{j}(k) \in R_{1}^{\prime \prime}
$$

is the vector of strategy parameters.

The object variable portion of an individual signifies the actual position in the real-valued search space for that individual. On the other hand, strategy parameters of an individual are responsible for mutation, and are often referred to as "step sizes".

At the $k^{1_{1}}$ generation, these $\mu$ individuals act as parents to produce an intermediate population $\left(P^{\prime}(k)\right)$ of $\lambda$ individuals $(1 \leq \mu \leq \lambda)$ hrough recombination. Usually, the ratio between $\mu$ and $\lambda$ is set to $1: 7$ [24]. When mating parents are chosen to generate offspring, they not only recombine their object variables but also their strategy parameters.

Definition 2. Recombination $\left(r_{\mathrm{BB} 1}\right)$

There are many recombination mechanisms used in ES. The most common ones are discrete recombination and intermediate recomhination. This paper employs binary discrete recombination, through which $\lambda$ offspring are created

$$
\vec{a}^{\prime}{ }_{j}(k)=r_{i, 1}\left(\vec{u}_{v}(k), \vec{a}_{1}(k)\right) ; \quad \forall j=1, \ldots, \lambda
$$

where $s$ and $t$ are the indices of two randomly drawn parents. Each component in $\vec{a}^{1}{ }_{i}(k)$ (objective variables as well as strategy parameters) is designated as the corresponding component of a chosen parent. This contributing parent is drawn randomly for each component of the offspring individual with replacement.

As mentioned earlier, there are many recombination operators employed by researchers. The algorithm proposed by this paper is applicable with any of these operators.

Once the intermediate offspring population of size $\lambda$ is obtained, it itself undergoes self-adaptive mutation. A standard implementation of $n$-dimensional self-adaptive mutation looks like the following:

Definition 3. Sielf-adaptive Mutation $\left(m_{\mathrm{SA}}\right)$

For each child in the offspring population, self-adaptive mutation is performed so that

$$
\ddot{a} "{ }_{i}(k)=m_{\mathrm{SA}}\left(\vec{u}^{\prime}{ }_{j}(k)\right) ; \quad \forall j=1, \ldots, \lambda
$$

where strategy parameters are varied first according to

$$
\left.\sigma^{\prime \prime}{ }_{i, i}(k)=\sigma^{\prime}{ }_{i, i}(k) \cdot \exp \mid \tau^{\prime} \cdot N_{i}(0.1)+\tau \cdot N_{j, i}(0,1)\right] ;
$$$$
V_{j}=1, \ldots, \lambda ; i=1, \ldots, n
$$

which are then used to mutate object variables

$$
\begin{array}{r}
x^{\prime \prime}{ }_{j, i}(k)=x^{\prime}{ }_{i, i}(k)+\sigma^{\prime \prime}{ }_{i,}(k) \cdot N_{i,}(0,1) ; \\
\forall j j=1 \ldots, \lambda ; i=1, \ldots, n
\end{array}
$$

In this paper, $\tau^{\prime}=(\sqrt{2 n})^{\prime}$ and $\tau=(\sqrt{2 \sqrt{n}})^{\prime}$, and $N(0,1)$ represents a normally distributed random variable with zero expectation and unity variance.

After mutation, the intermediate population $P^{\prime}(k)$ will enter the selection pool $\left(P^{* \prime}(k)\right)$ where a new population of $\mu$ individuals will be chosen as parents for the next generation $\left(I^{\prime}(k+1)\right)$. Conventionally, the selection pool can be either the entirety of offspring alone or the union of offspring and parents together [10], i.e.

Definition 4. C(OMMA LS and PLUS ES (i.e. $(\mu, \lambda)$ and $(\mu+\lambda) \mathrm{ES})$

In COMMA ES, the selection pool is made up of the entirety of $\lambda$ offspring alone

$$
P^{\prime \prime}(k)=\left\{\ddot{a}{ }_{1},(k), \cdots, \ddot{a}^{\prime \prime} ;(k)\right\}
$$

whereas in PLUS ES, the intermediate population consists of the union of $\mu$ parents and $\lambda$ offspring

$$
P^{\prime \prime}(k)=\left\{\vec{a}_{1}(k), \cdots, \vec{u}_{t \prime}(k), \vec{a} "_{1}(k), \cdots, \vec{a}{ }_{2},(k)\right\} \quad(10) .
$$

The COMMA and PLUS ES are special cases of the more general strategy of $(\mu, \kappa, \lambda) \mathrm{ES}$, where $\kappa$ denotes the maximal lifespan of an individual. With $\kappa=1$. the strategy resembles COMMA ES, while with $\kappa=\alpha$, it resembles PLUS ES [25].

Once pooled, these candidates are subject to the selection operator $\left(s_{T}\right)$ so that

$$
P(k+1)=s_{1}\left(P^{\prime \prime}(k)\right)
$$

Usually, truncation selection operator based on fitness ranking is used, although tournament selection and other selection operators could be used.

With these definitions, algorithm of the standard ES used in this paper are given as follows:

Algorithm I.Standard C'OMMA/PLUS ES

$$
k=0 \text {; }
$$

initializing $P(0)$ :

evaluating $P(0)$;

while (termination criteria is not fulfilled) do

$$
\begin{aligned}
& \text { recombination: } \ddot{a}^{\prime}{ }_{j}(k)==r_{i s}\left(\ddot{a}_{s}(k), \vec{a}_{t}(k)\right) \text {; } \\
& \forall \mathrm{j}=1, \ldots, \lambda \\
& \text { mutation: } \ddot{a}{ }_{i}(k)=m_{\mathrm{SA}}\left(\ddot{a}^{\prime}{ }_{j}(k)\right) ; \quad \forall \mathrm{j}=1, \ldots, \lambda \\
& \text { cvaluating } P^{\prime}(k)=\left\{\vec{a}^{\prime},(k), \cdots, \vec{a}^{\prime}{ }_{,}(k)\right\} \\
& \text { selection: } \\
& \text { if COMMA ES } \\
& P^{\prime \prime}(k)=\left\{a{ }^{\prime \prime},(k), \cdots, \vec{a}{ }_{k}(k)\right\} \text {; } \\
& \text { clse if PLUS ES } \\
& P^{\prime \prime}(k)=\left\{a_{1}(k), \cdots, \ddot{a}_{u}(k), \ddot{a} "_{1}(k), \cdots, \vec{a}{ }^{\prime}{ }_{k}(k)\right\} ; \\
& \text { ii }
\end{aligned}
$$




$$
\begin{array}{r}
\quad P(k+1)=k_{\mathrm{r}}\left(P^{\prime \prime}(k)\right) ; \\
k=k+1 ;
\end{array}
$$

\section{Improvements to Evolution Strategies}

\subsection{Previous Researches}

Because standard LS use deterministic truncation selection, whose selective pressure is high [13]. the population tends to search "locally" alter few generations [15]. Despite its simplicity and the high convergence rate it encourages, the truncation selection operator ironically undermines ES' capability to overcome local optima. Once the population is trapped, self-adaptation in mutation step sizes does nothing more than to aggravate the situation. Especially when the ratio between $\mu$ and $\lambda$ is very small, there exists such a crowding effect that only top-ranking individuals survive onto the next generation, whose strategy parameters are limited in size and often have great similarity. This phenomenon contributes to the fast convergence rate of COMMA ES.

There have been innumerable attempts to improve global performance of ES. Most of these improvements focus on explicitly replacing conventional crossover, mutation, and selection operators with new ones. In general, these efforts can be classified into two major categories. One attempts to deploy the population over search space with sufficient diversity. The other aims to enhance the efficacy of search guidance. These two approaches correspond to the two main concerns of all EC: "it can be argued that there are only two primary factors in genetic search ... population diversity and selective pressure. .. In a sense, this is just another variation on the idea of cxploration versus exploitation ..." [11]. However, due to the antipodal natures between exploitation and exploration, or between convergence and diversity, few of these improvements can fulfill both criteria simultaneously 112$][17][18]$.

There are two possible workabouts. The first is those algorithms that employ local competition on sub-population level instead of a global competition [16][19], because search diversity in these cases is upheld by spatially distinct sub-populations each evolving to local minima nearby. These modified algorithms often require a large population that will inevitably increase computation load. Moreover, the connection between global fitness landscape seen by the whole population and its collective mutation step sizes has been cut off. In fact. it can be asserted that these modified algorithms are equivalent to parallel search exercised with these sub-populations [20]. The other solution, which the authors try to exemplify in this paper, lies in altering the behavior of selection by making it more intelligent, more informed about the status quo of search process.

\subsection{Parental Population Sizing}

As mentioned earlier, one of the weaknesses in ES lies with the high selective pressure. Taken the fact that knowledge about fitness surface is encoded implicitly within spatial and fitness distribution of the population as well as distribution of mutation step sizes, much of these hints will definitely be eliminated under a high selective pressure. In this aspect, sustenance of population diversity is much more important than the creation of it through mutation. With the idea in mind, many studies have gone straight ahead to increase the size of the entire search population in order to host more diversity in one generation. However, sustenance of diversity lies not with sheer number of individuals. Having a large amount of similar individuals due to high selective pressures is worse than having only one in the population, because in the former case futile recombination and mutation are prone to happen.

Selective pressure in ES is determined by $\mu$ and $\lambda$, because it is a function of the ratio between size of the to-be-selected population (parents for the next generation) and size of the whole selection pool (for truncation selection operator) [13]. This ratio $T$ is inversely and nonlinearly proportional to selective pressure. In COMMA ES. $T$ is $(\mu)$ $\lambda$ ): in PLUS ES, $T$ is $(\mu /(\mu+\lambda))$. lor many researchers, it has been a conventional practice to use the recommendatory $\mu-\lambda$ ratio of $1: 7$ [6]. According to this suggestion, the selective pressure is high and premature convergence is almost inevitable.

The manipulation of selective pressure can be achieved through varying $\mu$ and $\lambda$. Nevertheless, absolute value of $\lambda$ also determines the computation load in terms of function evaluation required. Consequently, changing the value of $\mu$ seems to be a reasonable and efficient approach. Therefore, this paper proposes a modified ES algorithm that dynamically adjusts selective pressure through parental population sizing, in accordance with population diversity measures at each generation.

\section{Effects of Parental Population Sizes on ES}

To show the effects of $\mu r \lambda$ ratio has on ES. especially when $\mu$ varies over a fixed $\lambda$, several simulations are conducted over six benchmark functions.

\subsection{ES to be Compared}

The ES sets used in simulation are denoted by $\mu r \lambda$ COMMA/PLUS ES according to the actual number of individuals involved and the pooling scheme used. The sets employed are:
Set 1. 30-200 COMMA ES (30-200-CES), and 100-200 COMMA ES (100-200-CES), and 180-200 COMMA ES (180-200-C.ES).
Set 2. 30-200 PLUS ES (30-200-PES), and 100-200 PLUS ES (100-200-PES), and 180-200 PLUS ES (180-200-PES).

Other algorithm parameters are listed in Table 1.

\subsection{Benchmark Functions}

Task objectives in the simulation are function optimization, with fitness values ohtained directly from the objective 
Table 1. Parameters lsed for Simulation

\begin{tabular}{|c|c|c|}
\hline$\mu$ & $=$ as atemosed. & (number of parents) \\
\hline$\lambda$ & $=a s$ dithotith . & (number of oflspring) \\
\hline$\sigma^{i}(0)$ & $=3$. & (inilial step siz) \\
\hline$k_{\max }$ & $=20(00$. & $\begin{array}{l}\text { (number of generations. except } \\
\text { (0)(0) lor } / 6 \text { ). }\end{array}$ \\
\hline
\end{tabular}

functions. The benchmark functions are listed in Table 2 and $3[14][12][21]$. A few comments are worth mentioning beforehand. As can be observed in the tables, $f_{1}$ is the umimodal sphere minimization problem, while $f_{2}$ and $f_{3}$ are two different versions of the multimodal Ackley optimization problem with different local minimum basin widths and depths. In fact, these problems represent varying degrees of multimodality. For instance, $f_{f}$ is the Griewank function with the largest search space among the six benchmark problems. Finally, $f_{5}$ represents multimodal functions with many large local minima, whereas $f_{6}$ from the Shekel's family of lunctions has only ten local minima.

\subsection{Observation on Simulation Results}

Simulation results for Set $1 \& 2$ are tabulated in Table $4 \& 5$ respectively. Each simulation case has been conducted 50 times independently. After ranking individual results, the two top-most and two worst cases are discarded. The tabulated values are thus obtained from the central 46 cases. Best performing ES in each case is encircled with bold borders.

As can be seen from the results, optimal ratio between $\mu$ and $\lambda$ is indeed problem dependent. Nevertheless, both
COMMA and PLUS ES (Table 4 and 5) show a general preference for larger $11-\lambda$ ratios as the complexity (multimodality) of problems increases. Also noteworthy is the divergent inclination of "I $80-200-\mathrm{ClSS}$ " in Table 4. It is so because its harge $11-\lambda$ ratio produces such a low selective pressure that the population refuses 10 comverge.

\section{Dynamic Parental Population Sizing}

From the previous results, it can be concluded that conventional tr $\lambda$ ratio is not optimal for all problems. However, optimality of a given ratio is problem dependent. It seems that $11-\lambda$ ratio is indeed an important factor determining the performance of $E S$.

With these affirming results, the authors wish to pursue the idea of tuning $\mu-\lambda$ atio dynamically in the belief that (1) a excessively large $\mu$ value may still increase computation overhead, and (2) an intelligent sclection operator can reach a rasonable $\mu-\lambda$ ratio to maintain the balance between performance and algorithm resources.

The basic idea of dynamic parental population sizing is: when diversity of the population is high, lessen the selective pressure so that this diversity can be passed intact onto the next generation; on the other hand, when the diversity is low, choose only the topmost parent to save resources, and let self-adaptive mutation does its job. Here, diversity of the population is based on fitness only, since knowledge of the fitness terrain possessed by the $E \dot{S}$ is reflected in the fitness distribution of its population only. Therefore, several fitness

Table 2. Benchmark Functions lised in Simulation (wibh $n$ indicating dimension of the functions: i.e. $l \leq i \leq n$ )

\begin{tabular}{|c|c|c|}
\hline Function & II & Range \\
\hline$f_{1}(x)=\sum_{1} x$ & 30 & $\mid[-15,15]^{\prime \prime}$ \\
\hline$f_{i}(X)=(-10) \exp \left((-0.05) \sqrt{\frac{1}{\prime \prime} \sum_{i} x_{i}^{2}}\right) \cdots \operatorname{cop}\left(\frac{1}{n} \sum_{i} 1.3 \cos \left(0.5 \pi_{i}\right)\right)+20+0 / f \mathrm{set}_{3}$ & 15 & {$[1-15,15]^{\prime \prime}$} \\
\hline$\left.f_{1}(x)=\frac{1}{4000} \sum_{i} x_{i}=\prod \cos (y / f)+0\right) / f_{i} x_{1}$ & 30 & {$[-600,600]^{\prime \prime}$} \\
\hline $\begin{aligned} f_{s}(x)= & 0.02 \times \sum_{i}\left(x_{i}-2\right)^{2}+3.5 \sin \left(\left(\sum_{i} x_{i}\right)-5\right) \\
& +2.5 \sin \left(25 \cdot\left(\sum_{i} x_{i}-2.3 \cdot\left(\sum_{i} x_{i}\right)\right)+2.7\right)+1.5 \cos \left(10 \cdot\left(\sum_{i} x_{i}\right)-7.5\right)+\left(3 / f x^{\prime}\right.\end{aligned}$ & 15 & $|1-15,15|^{\prime \prime}$ \\
\hline
\end{tabular}

Table 3. Coefficient Constants Ised in $f_{6}(X)$ :

\begin{tabular}{|c|c|c|c|c|c|c|c|c|c|c|c|}
\hline \multicolumn{2}{|c|}{$j$} & 1 & 2 & 3 & 4 & 5 & 6 & 7 & 8 & 9 & 10 \\
\hline \multirow{4}{*}{$\vec{a}_{i}$} & $i=1$ & 4 & 1 & 8 & 6 & 3 & 2 & 5 & 8 & 6 & 7 \\
\hline & $i=2$ & 4 & 1 & 8 & 6 & 7 & 1) & 5 & 1 & 2 & 3.6 \\
\hline & $i=3$ & 4 & 1 & 8 & 6 & 3 & 2 & 3 & $\delta$ & 6 & 7 \\
\hline & $i=4$ & 4 & 1 & 8 & 6 & 7 & 9) & 3 & 1 & 2 & $\therefore 6$ \\
\hline \multicolumn{2}{|c|}{$c^{\prime} i$} & 0.1 & 0.2 & 0.2 & 0.7 & 0.4 & 61.6 & 03 & 0.7 & 0.5 & 0.5 \\
\hline
\end{tabular}


Table 4. (omparison among Results (COMMA LS (averaged over the central 46 out of 50 runs: "Mcin Best" indicating mean best fitness valuc in the last generation: "STD of Best" denoting standard deviation among the best litness values: top-ranking cases encircted by hold borders)

\begin{tabular}{|c|c|c|c|c|c|c|}
\hline \multirow{2}{*}{ Function } & \multicolumn{2}{|c|}{$3(1-200)-(\mathrm{l}) \mathrm{S}$} & \multicolumn{2}{|c|}{$10(0-200-C \mathrm{CS}$} & \multicolumn{2}{|c|}{$180-20(1-\mathrm{CTS}$} \\
\hline & Meim Besi & STH or lisest & Meim Best & STDOf Best & Meim Best & STO of BES \\
\hline i & $1.597 \times 10^{2}$ & $1110 \cdot 10^{2}=$ & $3.4860 \times 10^{-15}$ & $2.330 \times 10^{-13}$ & 10.5 .3 .020 & 1135,101 \\
\hline$f i$ & 0.076 & 0.251 & $1.756 \times 100^{3}$ & $2.182 \times 10^{-3}$ & 20.219 & 0.116 \\
\hline$f$ & 0.260 & $1126,-$ & $3.324 \times 16^{\circ}$ & $1.347 \times 10^{-1}$ & 1.114 & 4.120 \\
\hline fit & $0.0 \geq 6$ & 0.033 & 0.0011 & 01000.1 & 1908 & $(0.1): 44$ \\
\hline$f$ & 0.473 & 0.158 & 0.315 & 01.101 & 1.060 & 0.452 \\
\hline$f 6$ & -9.258 & $2.8(N)$ & -8.9013 & 2.920 & $-(1.252$ & 3.464 \\
\hline
\end{tabular}

diversity measures have to be defined first before going onto the modified algorithm.

Definition 5. Best, Average, Worst Population Finness $\left(F_{13}, F_{A}, F_{W}\right):$

Given a population $P^{\prime}(k)=\left\{\vec{a}_{i}(k) \ldots, \vec{a}_{t i}(k)\right\}$ of $\mu$ individuals with known litness values $\left\{\phi_{1}(k), \cdots, \phi \phi_{k}(k)\right\}=\left\{\left(\phi\left(\ddot{X}_{1}(k)\right), \cdots, \Phi\left(\ddot{X}_{\mu k}(k)\right)\right\}\right.$ at the $k^{\text {th }}$ generation over a minimization task, where $\Phi(\cdot)$ is the fitness function, the Average Population Fitness is computed by

$$
F_{\lambda}(k)=\frac{1}{\mu} \sum_{i} \phi_{j}(k)
$$

and the Best and Worst Population Fitness are defined respectively as

$$
F_{13}(k)=\min _{i}\left(\phi_{i}(k)\right)
$$

and

$$
F_{w^{\prime}}(k)=\max \left(\phi_{j}(k)\right)
$$

Definition 6. Finness Concentration $\left(F_{C}\right)$ :

With the same population given above, the measure of Fitness Concentration is defined to be

$$
F_{C}(k)=\frac{F_{W}(k)-F_{A}(k)}{F_{W}(k)-F_{B}(k)}
$$

however, if $F_{w}(k)$ equals $F_{13}(k), F_{C}(k)$ is defined as unity. Physical meaning of this measure is self-evident. When fitness distribution of the population is indistinctive, its average fitness (i.e. $F_{\lambda}(k)$ ) should be close to the worst population fitness $\left(F_{\mathrm{w}}(k)\right)$, and a low fitness concentration will be obtained. Contrarily, when most individuals of the population possess good fitness, a high fitness concentration is obtained. Moreover, given a unimodal landscape, a high fitness concentration value signifies that most individuals are all very close to the global optimum, whereas a low fitness concentration value means that only a few individuals make it to the optimum.

Based on this measure, a relation between the actual fitness landscape and fitness distribution of the population could be established. That is, a high $F_{C}$. suggests that fitness
Table 5. Comparison among Results (PLLUS ES) (averaged over the central 46 ott of 50 ) runs: "Mean Best" indicating mean

\begin{tabular}{|c|c|c|c|c|c|c|}
\hline \multirow{2}{*}{ Fuaction } & \multicolumn{2}{|c|}{ 3(1-201)-PES } & \multicolumn{2}{|c|}{ 10(1)-200-PNS } & \multicolumn{2}{|c|}{$180-200-\mathrm{PES}$} \\
\hline & Mein lest & STOU B Bes & Meam Best & ST'B of Best & Mean Best & STD of Best \\
\hline$f$ & 1. $664 \times 10^{-18}$ & $1.3999 \times 10^{-17}$ & $3(16,9) 100^{-13}$ & $1.19 n \times 10^{-t 3}$ & $3.413 \times 11^{-12}$ & $1.411 \times 10^{-11}$ \\
\hline is & 0.157 & 0.623 & $-1.229 \cdot 100^{\circ}$ & 1). $984: 16^{7}$ & $0.117 \times 10^{-7}$ & $1.323 \times 10^{-6}$ \\
\hline$f:$ & 19.5 .38 & $0.36,8$ & $3.321 \cdot 10^{\circ}$ & $(0.5)(x)$ & $3.324 \times 16^{-7}$ & $0.00 \%$ \\
\hline fit & 0.1179 & 0.152 & 0.600 .3 & 0.015 & $0.0(1)$ & 0.000 \\
\hline$f$ & 0.128 & 0.047 & 0.161 & 0.933 & $0.1(x)$ & 0.641 \\
\hline$d s$ & $-10.09 \%$ & 1.655 & -10.536 & $7.998 \div 10^{-100}$ & -10.5 .36 & $7.577 \times 10^{-16}$ \\
\hline
\end{tabular}
best fitness value in the last generation: "STO of Best" denoting standard deviation among the best fitness values: top-ranking cases encircled by bold borders)

distribution can somehow reflect the actual landscape, since fitness values of most individuals are concentrated. On the other hand. when $F_{f}$ is low, fitness landscape as seen by the population may be very different fiom the actual one, since representative tendency could not be established.

11 is known from the previous simulations that when selective pressure of this scheme is lowered, selective variance increases and divergence in COMMA ES will occur. This is because the truncation selection fraction $T$ is always smaller or equal to 0.5 in PLUS ES, while for COMMA ES, inappropriate ratio between $\mu$ and $\lambda$ will raise the fraction above 0.5 and bring about the inclination to diverge

Therefore, an extra lower bound $\left(p_{m}(k)\right)$ for the number of selection-pooling parents is devised. By this lower bound, there should be at least such many parents entering the selection pool to compete with the offspring. The modification means to make the truncation fraction $T$ less or equal to 0.5 , so that a reasonable degree of diversity can be remained without the threat of divergence $[13\}$. Hence this modified COMMA ES now turns into somewhat PLUS-like. This minimal number, $p_{\mathrm{m}}$, is defined as

Definition 7. Minimal Pooling Parent Number $\left(p_{m}(k)\right)$

For a given parental population size $\mu$,

$$
\begin{aligned}
& \rho_{11}(k)=\max ((2 \mid k(k)-\lambda), 1) \\
& \text { so that } T=\frac{\mu(k)}{\left(p_{m 1}(k)+\lambda\right)} \leq 0.5 .
\end{aligned}
$$

\subsection{Modified ES Algorithm}

According to the aforementioned rationale, the modified ES algorithm in terms of measures introduced is as follows:

Algorithm II. Modified ES with Dynamic Parental

Population Sizing (for minimization)

$k=0$;

initializing $P(0)$;

evaluating $P(0)$;

while (termination criteria is not fulfilled) do

$$
\text { recombination: } \begin{aligned}
\ddot{a}^{\prime}{ }_{j}(k)=r_{\mathrm{BD}}\left(\ddot{a}_{s}(k), \vec{a}_{i}(k)\right) ; \\
\forall \mathrm{j}=1, \ldots, \lambda
\end{aligned}
$$


Table 6. Comparison among Results (Moditied LS)

(averaged over the central 46 out of 50 ) runs: "Mean Best" indicating mean best fitness value in the last generation: "S"ID) of" Best" denoting standard deviation among the best litness values: top-ranking cases are encircled by toold horders)

\begin{tabular}{|c|c|c|c|c|c|c|c|c|}
\hline \multirow[t]{2}{*}{ Funclion } & \multicolumn{2}{|c|}{ Best of Set 1} & \multicolumn{2}{|c|}{$\begin{array}{c}\text { Modified } \\
\text { Comid ES }\end{array}$} & \multicolumn{2}{|c|}{ Best of Set 2} & \multicolumn{2}{|c|}{$\begin{array}{l}\text { Modilied } \\
\text { Plosiss }\end{array}$} \\
\hline & Nein 1sest & STD of Best & Mean Best & STI of Best & 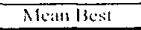 & S11D ol Best & Meim 1 Best & STI of kesl \\
\hline fi & $1.507 \times 10^{-27}$ & $1.140 \times 10^{-20}$ & $2.525 \times 10^{-47}$ & $1.597 \times 10^{-4 i}$ & $1.604 \times 10^{-15}$ & $1.399 \times 10^{-17}$ & $0.201 \times 10^{-21}$ & $3.153 \times 10^{-211}$ \\
\hline$f_{2}$ & $1.756 \times 10^{-7}$ & $2.182 \times 10^{-5}$ & $1.715 \times 10^{-7}$ & $6.286 \times 10^{-14}$ & $4.229 \times 10^{-7}$ & $9.984 \times 10^{-7}$ & $1.907 \times 10^{-7}$ & $1.053 \times 10^{-7}$ \\
\hline si & $3.324 \times 10^{-7}$ & $4.347 \times 10^{-1.3}$ & $3.324 \times 10^{-7}$ & $1.407 \times 10^{-15}$ & $3.324 \times 10^{-7}$ & 0.000 & $3.324 \times 10^{-7}$ & $1.692 \times 10^{-15}$ \\
\hline$f_{4}$ & 0.001 & 0.004 & 0.000 & 0.0012 & 0.000 & 0.000 & 0.0000 & 0.0012 \\
\hline$\%_{5}$ & 0.315 & 0.101 & 0.063 & 0.033 & 0.128 & 0.147 & 0.114 & 0.032 \\
\hline .6 & -9.258 & 2.800 & -10.536 & $8.460 \times 10^{-60}$ & -10.536 & $7.577 \times 10^{-16}$ & -10.536 & $5.005 \times 10^{-i 6}$ \\
\hline
\end{tabular}

muration: $\ddot{a}^{\prime \prime}{ }_{i}(k)=m_{\mathrm{S}_{\mathrm{i}}}\left(\ddot{a}^{\prime}{ }_{i}(k)\right) ; \quad \forall \mathrm{j}=1, \ldots, \lambda$

covbluaing $P^{\prime \prime}(k)=\left\{\ddot{a}^{\prime}(k), \cdots, a^{\prime},(k)\right\}$

calcularing Fitness Concentration $\left(F_{c}(k)\right)$ of the parental population $r(k)$

calculaing the new parental population size $\mu(k+1)$ by

$\mu(k+1)=(\lambda-1) !\left(1-F_{c}(k)\right)+1$

sielecion:

if COMMA ES

calculaing $/ p_{11}(k+1)$ from Eq. (16)

$\rho^{\prime \prime}(k)=\left\{\vec{a}_{1}(k), \cdots, \vec{c}_{p_{m 1}(k, 1)}(k)\right.$, $\vec{a}{ }^{\prime},(k), \cdots, \vec{a} ",(k) ?$

else if PLUS ES

$\rho^{\prime \prime}(k)=\left\{\ddot{a}_{1}(k), \cdots, \ddot{u}_{n}(k)\right.$.

fi

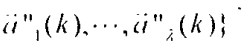

$P(k+1)=s_{\top}\left(P^{\prime \prime}(k)\right)$;

od

$k=k+1$.

\subsection{Performance of the Modified ES}

Performance of the modified ES in the same suite of benchmark functions is tabulated in Table 6 . If compared separately, it is obvious that the modified COMMA ES performs better than the original Set 1 ES in all problems. Also, the modified PLUS ES performs better than Set 2 ES in four out of six problems. Finally, when modified COMMA and PLUS ES are compared, it is found out that the former still performs better. It seems that due to the partial elitism of the modified COMMA ES and the parental population sizing scheme, the downsides of standard COMMA ES are all removed. Examples of the evolution of parental population size across the generations are given in Figure 2.

\section{Conclusions}

In this paper, the authors propose a modified algorithm to calculate parental population size for ES. The idea comes from the observation that conventional $\mu-\lambda$ ratio used in ES produces an excessively high selective pressure in the Iruncation selection operator for population diversity to sustain. Therefore, premature convergence occurs under the disguise of a fast convergence velocity. This is also why ES have been called by many as a local optimization technique. Some reasoning on the tension between diversity and selective pressure in ES can be found in [22][23].

It is concluded by the authors that high selective pressure of ES ranking-based selection operator hinders free evolution of self-adaptation by admitting only a small portion of the best of offspring to survive as parents in the next generation. These new parents often have similar strategy parameters in addition to similar object variables. Moreover, since unsupervised self-adaptation of strategy parameters fails to readjust mutation steps in accordance with eurrent search status. mutated individuals have difficulties in overcoming local optima. Consequently, when these two deficiencies add up, a vicious circle emerges: lack of diversity in mutation steps leads to homogeneity in object variables, which in turn aggravates the irrelevancy of mutation operator

To solve this deficiency, the mechanism of parental population sizing is proposed. The mechanism feeds on diversity measures of the parental population and computes the number of parents to be selected for the next generation, adjusting selective pressure dynamically. By varying the number of parents over a fixed offspring population size, diversity in mutation steps is preserved and deficiency repaired. What is more, since only the size of parental population is altered instead of that of offspring population, there should be less computation overhead and memory requirement than conventional offspring population sizing techniques. To sum up, the modification proposed in this paper implies more efficient and relevant usage of population resources.

Moreover, a phenomenon is found that parental population sizes in some cases fall down to the lowest level of one and stays there for the rest of simulation. This interesting point suggests (1) a convergent state is reached and external verification mechanism should step in, or (2) 


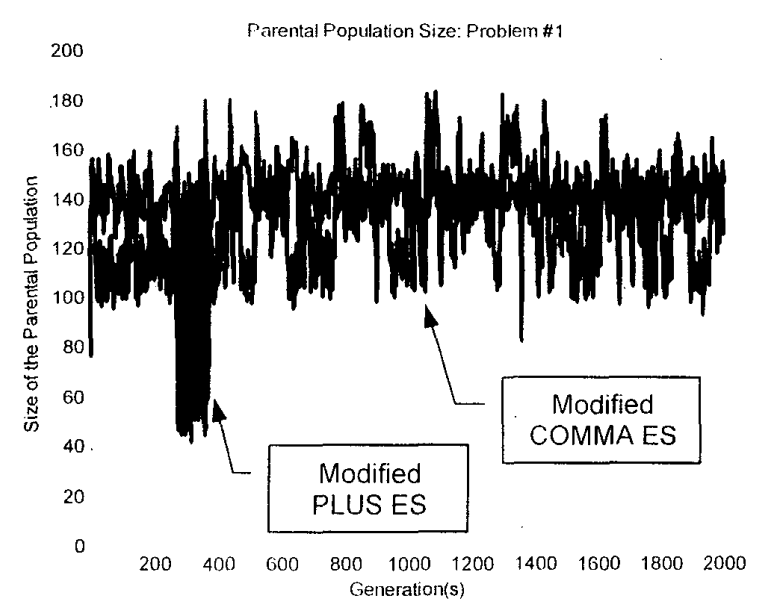

(a)

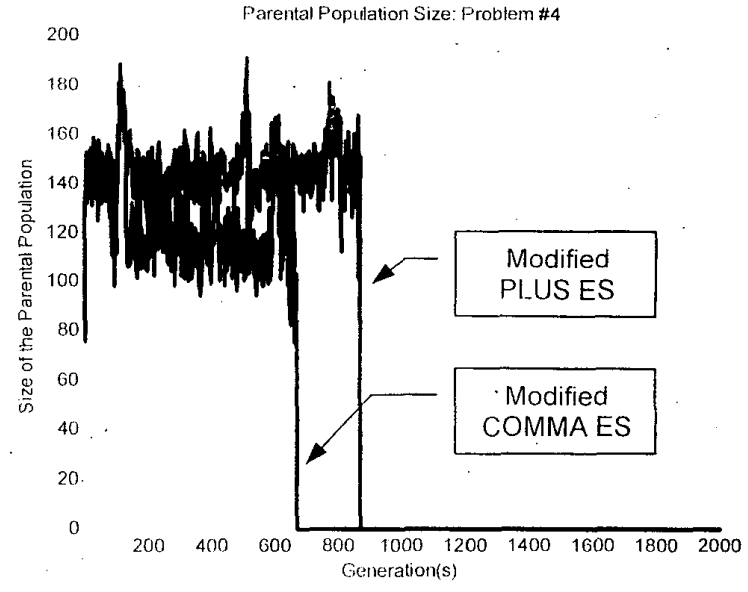

(b)

ligure 2. Exemplary ligure of Parental Population Size (averaged over the central 46 runs: 2.a-- for $/ 1$ and 2 .b-- for $/ 4$ )

further modification of the parental population sizing approach (for example, the lowest level of parental population number should be two rather than one). At any rate, simulation on several benchmark problems vindicates the virtue of this population sizing approach.

\section{References}

[1] I. Rechenberg. Evolutionsstrategie: Optimierung technischer Systeme nach Prinzipien der biologischen Evolution. Stuttgart, Germany: Frommann-Holzboog, 1973.

[2] H.-P. Schwefel, Evolutionsstrategie und numerische Optimierung, Dissertation, Technische Universität Berlin, Germany, May 1975.

[3] J. H. Holland, "Outline for a Logical Theory of Adaptive Systems", J. Assoc. Comput. Mach., vol. 3, pp. 297-314, 1962.

[4] L. J. Fogel, "Autonomous Automata", Ind. Res., vol. 4 , pp. 14-19, 1962.

[5] H.-P. Schwefel and F. Kursawe, "On Natural Life's Tricks to Survive and Evolve", Proceedings of The 1998 IEEE International Conference on Evolutionary Computation, pp. 1-8, 1998.

[6] T. Bäck, U. Hammel, and H.-P. Schwefel, "Evolutionary Computation: Comments on the History and Current State", IEEE Transactions on Evolutionary Computation, vol. 1, no. 1, pp. 3-17, Apr. 1997.

[7] D. B. Fogel, "An Introduction to Simulated Evolutionary Optimization", IEEE Trans. on Neural Networks, Vol. 5, No. I, pp. 3-14, 1994.

[8] T. Bäck, "On the Behavior of Evolutionary Computation in Dynamic Environments", Proc. 1998 IEEE World Congress on Computational Intelligence. pp. $446-451,1998$
[9] J. Born, Evolutionsstrategien zur Numerischen Lösung von Adaptationsaufgaben. Dissertation A, Humcboldt-Universität, Berlin. 1978.

[10] Jörg Heitkötter and David Beasley, eds., "The Hitch-Hiker's Guide to Evolutionary Computation: A list of Frequently Asked Questions (FAQ)", IJSENET: compai.genetic. Available via inonymous FTP from rtfm.mit.edu/pub/usenet/news.answers/ai-faq/genetic/, 1999.

[1I] D. Whitley, "The GENITOR Algorithm and Selection Pressurc: Why Rank-Based Allocation of Reproductive Trials is Best", Proceedings of the Third International Conference on Cienetic Algorithms, San Mateo: Morgan Kaufmann. pp.116-121, 1989.

[12] X. Yao and Y. Liu, "Evolutionary Programming Made Faster". IEEE Trans. Evolutionary Computation, vol. 3, no. 2, July 1999 .

[1.3] T. Blickle and L. Thiele, "A Comparison of Selection Scheme Used in Evolutionary Computation", Evolutionary Computation. vol. 4, no. 4, p.p. 361-394, 1097.

[14] T. Bäck and H..P. Schwefel, "An Overview of Evolutionary Computation for Parameter Optimization", Evolutionary Computation, vol. 1, no. 1, pp. 1-23, 1993.

[15] H.-G. Beyer, "Toward a Theory of Evolution Strategies: Self-Adaptation", Evolutionary Computation, vol. 3, no. 3, pp. 311-347, 1996.

[16] M. Gorges-Schleuter, "A Comparative Study of Global and Local Selection in Evolution Strategies", PPSN V, pp. 367-377, 1998

[17] I. Rechenberg, Evolutionsstrategie '94, Stuttgart: Frommann-Holzboog, 1994.

[18] A. Ostermedier, A. (iawelczyk, and N. Hansen, "A Derandomized Approach to Self-Adaptation of 
Evolution Strategies", Evolutionary Computation, vol. 2, no. 4, pp. 3(69-380. 1995.

[19] J.-M. Yang. Y.-P. Chen. J.-T. Hong, and C.-Y. Kao, "Applying Fanily (ompetition to Evolution Strategies for Constrained Optimization", Lecture Noles In Computer Science, Issue 1213, pp. 201-211, 1997.

[20] H. Mühlenbein. "Asynchronous Parallel Search by the Parallel Genetic Algorithm", Proceedings of the Third IEEE Symposium on Parallel and Distributed Processing, pp. 526-533, 1991.

[21] Olivier François, "An Evolutionary Strategy for Global Minimization and Its Markov Chain Analysis", IEEE Transactions on Evolutionary Computation. Vol. 2, No. 3, pp. 77-90, 1998.

[22] Hans-Georg Beyer. "Toward a Theory of Evolution Strategies: On the Benefits of $\operatorname{Sex} \cdots$ the $(\mu / \mu . \lambda)$ Theory". I:volutionary Computation, Vol.3 No, 1, pp. $81-111,1090$.

[23] Hans-Geor Beyer, "Mutate Large, But Inherit Small! On the Analysis of Rescaled Mutations in $(1, \lambda)-\mathrm{ES}$ with Noisy litness Data", PPSN V, LNCS no. 1498 , pp. 109-118, 1998 .

[24] H.-P. Schwefel, "Collective Phenomena in Evolutionary Systems", Preprints of the 31 st Annual Meeting of the International Society for General System Research. Budapest, 2, pp. 1025-1033, 1987.

[25] H.-P. Schwefel and Günter Rudolph, "Contemporary Evolution Strategies". Advances in Artificial Life, LNAI 929, pp. 893-907, 1995. 